\title{
Aa. Vv., «George Sand Studies», t. XXVII
}

\section{Morena Petrich}

\section{(2) OpenEdition}

\section{Journals}

\section{Edizione digitale}

URL: http://journals.openedition.org/studifrancesi/7297

DOI: $10.4000 /$ studifrancesi.7297

ISSN: 2421-5856

\section{Editore}

Rosenberg \& Sellier

\section{Edizione cartacea}

Data di pubblicazione: 1 avril 2010

Paginazione: 171-172

ISSN: 0039-2944

\section{Notizia bibliografica digitale}

Morena Petrich, «Aa. VV., «George Sand Studies», t. XXVII», Studi Francesi [Online], 160 (LIV | I) | 2010, online dal 30 novembre 2015, consultato il 08 janvier 2021. URL: http://journals.openedition.org/ studifrancesi/7297; DOI: https://doi.org/10.4000/studifrancesi.7297

Questo documento è stato generato automaticamente il 8 janvier 2021.

\section{(c) $($ ) $(9)$}

Studi Francesi è distribuita con Licenza Creative Commons Attribuzione - Non commerciale - Non opere derivate 4.0 Internazionale. 


\title{
Aa. Vv., «George Sand Studies», t. XXVII
}

\author{
Morena Petrich
}

\section{NOTIZIA}

«George Sand Studies», t. XXVII, 2008, pp. 113.

1 L'ultimo numero della rivista «George Sand Studies» è dedicato al teatro sandiano, ricco e complesso universo spesso trascurato da critica e lettori.

2 Il primo contributo, di Anne-Marie BARON ( $\mathrm{La}$ critique religieuse dans "Spiridion" et "Mademoiselle de la Quintinie": du roman à thèse au théâtre à thèse, pp. 4-11), ripercorre le peculiarità e le tappe evolutive che caratterizzano la trasposizione scenica di romanzi di Sand ed in particolare del testo, fortemente anticlericale, del 1863, Mademoiselle de la Quintinie, che diventa "pièce» teatrale nel 1872 e viene rappresentato una sola volta nel 1888. L'autrice insiste sulla valenza altamente pedagogica del teatro sandiano, che diviene uno strumento eminentemente didattico, e sul messaggio moralizzatore che pervade sia il testo narrativo in oggetto che il suo rifacimento drammatico e ne fa un accorato appello ad un ritorno alla purezza del messaggio evangelico. Olivier BARA ("Maître Favilla", ou le théâtre à la folie: illusion et hallucination, pp. 12-25) conduce invece un'interessante riflessione su un'altra modalità di declinazione del Bene all'interno dell'opera di Sand, incentrata sull'idealismo di impianto platonico che indirizza la creazione artistica verso la «manifestazione» e l'illustrazione del mondo come dovrebbe essere. L'arte come nuova catechesi e l'artista come vate e salvatore trovano nel sogno e nell'illusione l'effigie ed il manifesto dell'utopia da comunicare al popolo. La vertigine allucinatoria e la follia che minacciano il protagonista di Maitre Favilla pongono l'interrogativo sulla spesso vilipesa lucidità dello slancio idealista e sulla sua importanza: l'autore mette in evidenza come Sand, attraverso numerose e minuziose didascalie, abbia prestato grande cura ai dettagli scenici e scenografici al fine di assicurare una perfetta convergenza empatica tra l'attore - catalizzatore e medium - e 
lo spettatore - discente. Solo così, grazie ad una concertata mimesi, avrebbe potuto realizzarsi il flusso diretto della parola rivelatrice e profetica.

3 L'impegno e le convizioni di Sand circa la necessaria emancipazione femminile dominano tre testi che vengono analizzati da Catherine MASSON nel suo contributo su Féminisation et subversion du héros romantique dans le théâtre de George Sand (pp. 26-38). L'autrice illustra come nel decennio 1831-40, sperimentando con i drammi Une Conspiration en 1537, Gabriel e Cosima una scrittura teatrale in personale chiave romantica, Sand denuncia l'esclusione sociale della donna e la violenza dell'impianto patriarcale. Dopo l'ultima «pièce», rivelatasi un doloroso insuccesso, si dovranno attendere otto anni perché gli eventi rivoluzionari del 1848 facciano tornare sulle scene parigine un'altra opera teatrale sandiana, Le Roi attend, seguita, tre anni dopo, dal dramma Claudie, che rappresenta l'ultimo anello dell'evoluzione femminile (femminista) dell'eroe romantico e la riaccesa fede in un reale cambiamento della società. È ancora su quest'ultima pièce che porta la sua attenzione Dominique LAPORTE ("Claudie", ou les avantages de la comédie sérieuse: de 'l'inconduite' parodique ("Claudine") à la reconduction intratextuelle ("Le Pressoir", "Les Don Juan de Village"), pp. 39-62) : partendo da una disamina della lacerazioni e delle conflittualità tra le opere ed il pensiero di Sand ed il contesto politico e culturale del Secondo Impero e della Terza Repubblica, l'autore indaga le ragioni e le ripercussioni del successo del dramma Claudie del 1851 alla luce della sua parodia Claudine, ou les avantages de l'inconduite. Stabilendo un parallelo e un confronto tra l'originale e la replica parodistica, anche con l'ausilio degli strumenti di indagine mutuati da Gérard Genette, e approdando a Le Pressoir e Les Don Juan de village, interpretati rispettivamente come «filiazione» e «replica carnavalesca» alla parodia di Siraudin e Rousseau de Beauplan, l'autore pone in debito rilievo la componente politico-satirica della produzione teatrale sandiana e il suo interesse dal punto di vista sociologico.

4 Nicole LUCE dedica il suo contributo ("François le champi": représentation théâtrale et critique dramatique, pp.63-76) all'enorme, inatteso successo riscosso dalla versione scenica di François le champi, che, con le sue 140 rappresentazioni e varie riprese, testimonia dell'apprezzamento del pubblico per le «pièces» di Sand, a cui ha sempre fatto eco l'adesione della critica. L'autrice enfatizza i termini della portata rivoluzionaria di questa commedia che, con l'ardita scelta della forma espressiva dialettale («avec la création d'un idiome franco-berrichon»), e un «décor» che riproduce fedelmente, quasi con rigore etnografico, il contesto socio-culturale dei protagonisti, inaugura una nuova maniera di fare teatro. La pionieristica frontiera della rappresentazione scenica così intrapresa trova corrispondenza e sostegno nel profondo rinnovamento del linguaggio e della condizione dell'attore di Sand: Shira MALKIN (L'École et la troupe: aspects de la pédagogie de George Sand et de Jacques Copeau, pp. 77-94) ricorda come non solo la sua produzione teorica si orienti verso la celebrazione dell'improvvisazione ma come anche la stessa pratica privilegiata in venti anni di spettacolo a Nohant ne ricalchi quegli intenti riformatori, che sono enucleati in particolare ne Le Château des désertes e Masques et bouffons (quest'ultimo scritto in collaborazione con il figlio Maurice). L'autrice illustra l'influenza di tale pensiero sull'elaborazione concettuale del drammaturgo Jacques Copeau, uno dei padri del teatro moderno e fondatore del Théatre du Vieux Colombier a Parigi. È principalmente nella concezione avanguardista della troupe come cellula comunitaria, che accomuna l'estetica di Sand e di Copeau, che risiede il punto di rottura più forte del loro 
rinnovamento che, come lo esplicita l'autrice, celebra una nuova «dimensione famigliare, etica ed estetica» dell'attore e della compagnia teatrale. Nell'ultimo articolo, À propos d'“Anzoleto" (pp. 95-105), Michèle HECQUET indaga il ruolo del personaggio che compare sia in Consuelo che ne La Comtesse de Rudolstadt, e che, dietro l'ingannevole apparenza del nome angelico, rivela une personalità che lo avvicina - per l'incostanza, l'ebbrezza della menzogna e del tradimento, non da ultimo, la vertigine della sopraffazione - a Don Giovanni e al Diavolo stesso.

5 La varietà e la ricchezza dei contributi fanno di questo numero della rivista un'ottima e stimolante lettura per avvicinare appassionati e neofiti al teatro di Sand. 